\title{
Indicação de tomografia computadorizada de feixe cônico para pacientes infantis
}

\author{
Indication of cone-beam computed tomography for children's patients
}

Recebido: 01/10/2021 | Revisado: 06/10/2021 |Aceito: 09/10/2021 | Publicado: 12/10/2021

\author{
Karla Ferreira da Silva \\ Universidade Brasil, Brasil \\ Email: Kferreira05@gmail.com \\ Marcus Vinícius Sales Arrais de Carvalho \\ Universidade Brasil, Brasil \\ Email: marcos.vi1618@gmail.com \\ Paulo Roberto Gromatzky \\ Universidade Brasil, Brasil \\ E-mail: pgromatzky@gmail.com
}

\begin{abstract}
Resumo
A tomografia computadorizada de feixe cônico é um método de diagnóstico inovador que progrediu na odontologia moderna sendo adequada para quase todas as áreas da odontologia incluindo a odontopediatria, usado para analisar e descrever processos patológicos, visualizar dentes impactados, avaliar seios da face, diagnosticar traumas e mostrar a composição óssea da articulação temporomandibular. O exame de imagem é de grande valor no diagnóstico complementar, proporcionando dados importantes para o sucesso do tratamento odontológico pediátrico, possibilitando aplicação de uma técnica menos invasiva. Projetado para trazer a aplicabilidade clínica da tomografia computadorizada em odontopediatria para dentistas, investigou alguns casos de uso desta tecnologia de imagem. O objetivo deste trabalho foi realizar uma revisão de literatura citando o uso e as indicações da tomografia computadorizada de feixe cônico na odontopediatria. Na metodologia foram consideradas 38 publicações entre 1992 a 2020, com base em dados inglês e português: Google Acadêmico, PubMed, Scielo (Scientific Eletronic Library Online) e BDTD (Biblioteca digital brasileira de tese e dissertações). Concluímos que a tomografia computadorizada de feixe cônico é um exame imagiológico importante para o cirurgião-dentista, pois auxilia no diagnóstico de lesões, traumas, detecção de processos patológicos, planejamentos cirúrgicos, síndromes genéticas entre outros . Palavras-chave: Odontopediatria; Tomografia computadorizada; Odontologia.
\end{abstract}

\begin{abstract}
Cone-Beam Computed Tomography is na innovative diagnostic method that has progressed in modern dentistry and is suitable for almost all areas of dentistry including pediatric dentistry, used to analyze and describe pathological processes, visualize impacted teeth, evaluate sinuses, diagnose trauma, and show the bone composition of the temporomandibular joint. The imaging exam is of great value in complementary diagnosis, providing important data for the sucesso of pediatric dental treatment, allowing the application of a less invasive technique. Designed to bring the clinical applicability of computed tomography in pediatric dentistry to dentists, investigated some cases of use of this imaging technology. The objective of this work was to perform a literature review citing the use and indications of cone beam computed tomography in pediatric dentistry. The methodology considered 38 publications from 1992 to 2020, based on English na Portuguese data: Google Academic, PubMed, Scielo (Scientific Eletronic Library Online) and BDTD (Brazilian Digital Library of Theses and Dissertations. We conclude that cone beam computed tomography is an important imaging exam for the dental surgeon, because it helps in the diagnosis of injuries, trauma, detection of pathological processes, surgical planning, genetic syndromes, among others.
\end{abstract}

Keyswords: Pediatric dentistry; Computed tomography; Dentistry.

\section{Introdução}

Os exames radiológicos são extremamente essências para a odontologia e podem auxiliar no desenvolvimento de planos de tratamento. A tomografia computadorizada de feixe cônico é um dos meios de diagnóstico que nos permite a avaliação tridimensional de uma estrutura. Em odontopediatria, a tomografia é muito importante porque nos permite observar mudanças precoces no desenvolvimento de anormalidades, erupções, patologias, traumas e cáries, avaliar os seios da face e diagnosticar traumas. Como todo método radiológico, a tomografia computadorizada tem suas limitações e deficiências, como alto custo do exame, alta dose de radiação ao paciente e possível movimentação durante o exame causando um longo tempo de 
exposição. Uma vez apontados, os benefícios ao paciente, podem trazer uma sensação de segurança aos profissionais e aos pacientes, justificando as limitações da tomografia.

O objetivo deste trabalho visa entender a importância da tomográfica computadorizada de feixe cônico no diagnóstico, suas limitações e seus achados clínicos conforme a literatura.

\section{Metodologia}

Por meio de uma revisão de literatura que foi abordado o tema indicação da tomografia computadorizada de feixe cônico para pacientes infantis na qual será abordado um estudo descritivo, cujo objetivo é compreender os conhecimentos e as condutas em relação à tomografia computadorizada de feixe cônico na odontopediatria. Os descritores utilizados para busca foram: tomografia computadorizada na odontopediatria, tomografia computadorizada de feixe cônico na odontopediatria, tomografia cone beam, trauma dental, dentes impactados, planejamento ortodôntico, condições patológicas. Para esta revisão foram selecionados 38 publicações que se enquadram nos critérios de inclusão e exclusão. Koche, J. C. (2011).

Os critérios de inclusão foram os seguintes: artigos e revistas, disponível nos idiomas em língua inglesa e portuguesa nos site como: Google Acadêmico, PubMed, Scielo (Scientific Eletronic Library Online) e BDTD (Biblioteca digital brasileira de tese e dissertações), contextos disponíveis em meios eletrônicos, que foram publicados entre 1992 a 2020 . Os critérios de exclusão: relatórios e publicações sem fontes confiáveis, materiais com disponibilidade incompleta que não abordava com clareza o tema.

\section{Revisão da Literatura}

\section{Tomografia convencional}

Tomografia convencional é considerada uma técnica que fornece imagens de uma ou mais partes da estrutura de interesse, enquanto as estruturas acima ou abaixo da área aparecem desfocadas. As imagens dessas estruturas são como se nelas fossem realizados vários cortes, em vários planos de espessura relativamente pequena. É uma técnica muito útil quando é necessária a obtenção de imagens das estruturas anatômicas sobrepostas (BERNI et al., 2003).

Uso de tomografia convencional faz uso do filme e de fontes de radiação para criar partes do objeto. Por meio desses movimentos, a tomografia elimina o problema de sobreposição (ABRAMOVITCH, 1995).

\section{Tomografia computadorizada convencional}

Comparada com a radiografia convencional, a tomografia computadorizada apresenta vantagens importantes: em primeiro lugar, a informação tridimensional é apresentada na forma de uma sequência de cortes finos da estrutura interna na parte estudada. Uma vez que o feixe de raios é estritamente colimado para este corte em particular, as informações obtidas não serão sobrepostas pela estrutura anatômica sobreposta, nem serão degradadas pela radiação secundária e difusa do tecido fora de corte em estudo. Em segundo lugar, em comparação com a radiografia tradicional, é mais sensível na distinção de tipos de tecidos, de modo que as diferenças entre os tipos de tecidos podem ser descritas e estudadas com mais clareza. Em terceiro lugar, a facilidade de manusear e regular após ter sido finalizada a varredura da tomada (BONTRAGER, 2003).

A tomografia computadorizada de feixe cônico (TCFC) foi incorporada na Odontologia em 1998, na Europa, aprovada para uso apenas em 2001 nos Estados Unidos (EUA). Em setembro de 2002 em Pacific Grove, Califórnia, EUA, iniciou-se uma análise sobre um futuro impacto na Odontologia causado por essa tecnologia discutida pela primeira vez no congresso: "Imagiologia Craniofacial no Século XXI". Desde aquele momento, a tomografia computadorizada de feixe cônico 
recebeu um rápido progresso, tornando-se exigida em diversas áreas por diversas especialidades para imagens em três dimensões (3D) nítidas, resolutas e precisas (KAPILA et al., 2015).

A tomografia computadorizada utilizada, notadamente, na odontologia é atualmente conhecida como tomografia computadorizada volumétrica ou de feixe cônico (cone beam), equivale o desenvolvimento de um tomógrafo de baixo custo, indicada para regiões de cabeça e pescoço. Essa tecnologia permite uma reprodução de imagens em 3D (imagens tridimensionais), podendo-se obter uma melhor qualidade dos tecidos orofaciais, havendo uma mínima distorção possível e com uma menor dose de radiação em comparação à TC convencional. Desde a inclusão da TCFC na odontologia, seu uso tem aumentado significadamente (CARRASCO et al., 2018).

Em 1981, o Comitê Ad Hoc Committee on Pedodontic Radiology desenvolveu orientações no exame radiográfico de pacientes odontológicos pediátricos. Seis anos depois, a Food and Drug Administration (FDA) dos Estados Unidos publicou recomendações desenvolvidas por um painel dentário de especialistas, que incluía um representante da AAPD, se reunindo "para chegar a um consenso sobre a padronização de procedimentos radiográficos odontológicos". Em 2002, a American Dental Association (ADA) iniciou uma revisão desse documento (American Academy of Pediatric Dentistry; 2020).

A principal vantagem da Tomografia computadorizada é que ela forneça um conjunto de dados em escala real com seções transversais multiplanares (planos axial, sagital e coronal) e reconstruções tridimensionais, produzidos por uma varredura (APS, 2013).

Em relação à Técnica da Tomografia Computadorizada Cone Beam, possui uma diferença maior das outras técnicas radiográficas, pode-se obter uma maior precisão no diagnóstico da localização e extensão de lesões, uma das técnicas mais utilizadas como exame complementar em determinados casos clínicos. Esta técnica possibilita uma melhor visualização das estruturas anatômicas (BeRNARDES, 2008; OLMEZA et al., 2011).

A Tomografia computadorizada de feixe cônico é ainda proeminente na odontopediatria porque pode detectar precocemente alterações no desenvolvimento, erupção dentária, anormalidades e patologias. Além de verificar a relação entre o germe dentário permanente e os elementos dentais decíduos, um dos principais usos da tomografia computadorizada em odontopediatria é detectar a presença de impactação de elementos dentais. Como todo método radiológico, a tomografia computadorizada tem suas limitações e deficiências, como alto custo do exame, longo tempo de exposição e, portanto, alta dose de radiação ao paciente e possível movimentação durante o exame (BARBOSA et al., 2018).

\section{Princípios básicos de proteção contra radiação}

De acordo com APS (2013, p.132), referente às crianças, o cirurgião-dentista deve ter uma atenção maior para não expor nenhuma às radiações desnecessárias afirma:

No que diz respeito às crianças, o dentista deve ser ainda mais alerta para não expor qualquer indivíduo jovem em crescimento à radiação "desnecessária". Fins econômicos nunca deve ser uma razão para submeter os pacientes à radiação ionizante. (tradução nós)

Os objetivos econômicos nunca devem ser um motivo para os pacientes receberem radiação ionizante. Isso nos leva a três princípios básicos de proteção contra radiação. Em primeiro lugar, existe o "princípio da legitimidade", que significa que as radiografias só são apresentadas quando não existe outra forma de obter as informações necessárias. Se o paciente não conseguir completar a operação, não devem ser feitas as radiografias, por exemplo, se a criança não conseguir ficar imóvel por tempo suficiente durante as radiografias, o cirurgião dentista não deve prosseguir com o procedimento (APS, 2013). 


\section{Dose de radiação}

De acordo com APS (2013, p.132), ainda é pouco falado sobre dose de radiação na literatura afirma:

Na literatura, infelizmente, nem sempre está claro o que se entende por dose de radiação, portanto, alguma explicação é necessária. A “dose de radiação absorvida” (abreviada como D) é a medida da quantidade de energia absorvida do feixe de radiação por unidade de massa de tecido; a unidade é o Gray. (tradução nós)

\section{Prescrição necessária}

De acordo com American Dental Association (2012, p. 901), os cirurgiões dentistas devem usar julgamentos éticos e cautelosos para a prescrição da TCFC, observando o contexto clínico específico e as necessidades de cada paciente:

Os cirurgiões dentistas devem usar o julgamento profissional para a prescrição de exames de TCFC, consultando as recomendações das diretrizes disponíveis, considerando a situação clínica específica e as necessidades de cada paciente. (tradução nós)

\section{Resultados de acordo com o contexto clínico}

\section{Cárie}

De acordo com Horner et al., (2020, p. 421), menciona que as evidências relacionadas à TCFC e ao diagnóstico de cárie baseavam-se predominantemente em pesquisas e a maioria dos estudos mostrou pouca diferença na precisão do diagnóstico quando a imagem CBCT foi usada em comparação com a radiografia intraoral afirma:

As evidências relacionadas à TCFC e ao diagnóstico de cárie baseavam-se predominantemente em pesquisas ex vivo e a maioria dos estudos mostrou pouca diferença na precisão do diagnóstico quando a imagem CBCT foi usada em comparação com a radiografia intraoral. (tradução nós)

\section{Reabsorções radiculares}

Recentes estudos indicam que a tomografia computadorizada de feixe cônico pode ser utilizada com resultados satisfatórios no diagnóstico de reabsorções radiculares de dentes permanentes (PATEL ET al., 2007; KAMBUROGLU et al., 2008; LIEDKE et al., 2009; DURACK et al., 2010; KUMAR et al., 2011).

\section{Dentes impactados}

Serve como exame complementar de diagnóstico para elaborar um plano de tratamento de caninos impactados (Haney et al. 2010).

O trabalho de Katheria et al. (2010), cita que a TCFC está sendo apresentado como seguro para a correta localização dos dentes impactados, identificação de reabsorções radiculares e para a formação de um plano de tratamento. A tomografia computadorizada de feixe cônico tem se mostrado um exame complementar importante e preciso para diagnóstico e o planejamento cirúrgico de dentes retidos (PRIMO et al. 2011).

\section{Trauma dentário}

Para dentes não tratados endodonticamente, a TCFC pode levar a precisões diagnósticas muito altas paras fraturas radiculares. Além disso, esses níveis de exatidão são maiores do que quando utilizamos radiografias periapicais (HORNER et al., 2020). Alguns estudos indicam a TCFC como método mais indicado para detecções de fraturas radiculares verticais (MANSINI et al., 2010). 
Caso não aja a possibilidade ou dúvidas na identificação das fraturas radiculares por meio das radiografias convencionas, a tomografia computadorizada de feixe cônico pode ser indicada, pois, proporciona ao cirurgião dentista a obtenção de imagens em três dimensões de alta qualidade melhorando a visualização e a localização da extensão da fratura radicular (VIDIGAL et al., 2014).

\section{Condições patológicas}

Há uma falta completa de evidências pediátricas para o uso CBCT em doenças periodontais. A partir das evidências derivadas de estudos clínicos em adultos, a CBCT só seria indicada para casos excepcionais que requerem manejo complexo, por exemplo, em cirurgia periodontal regenerativa no contexto pediátrico, isso seria extremamente raro (HORNER et al., 2020). A tomografia computadorizada é útil na avaliação das lesões de cabeça e pescoço, avalia a presença ou extensão dos tumores envolvidos na maxila, ou na mandíbula, infecção ou outra patologia (KING et al., 1992).

Não há evidências de que a infecção dentária aguda seja uma indicação para TCFC e nenhuma orientação relevante foi encontrada. Evidências da revisão de estudos sistemáticos mostram que, para cavidades ósseas periapicais preparadas mecanicamente ou quimicamente, o uso de TCFC pode fornecer uma maior precisão diagnóstica do que os exames de raios-X tradicionais (HORNER et al., 2020). Auxilia no diagnóstico de implicações endodônticas (D’ ADDAZIO et al. 2010).

A tomografia auxilia no processo de reconhecimento patológico e permite sua reconstrução em três tamanhos, ajuda o cirurgião dentista a traçar um plano de tratamento. Permite a detecção do conteúdo do espaço patológico como, por exemplo, sangue, lesões císticas, tumores antes mesmo de realizar uma cirurgia invasiva. A tomografia pode ser usada para identificar perfuração cortical ou invasão de tecidos moles adjacentes e pode registrar linfonodos regionais no caso de estadiamento de tumor maligno (PARKS et al., 2000). Auxilia na localização de cistos odontogênicos e corpos estranhos (ABRAHAMS, 1993).

As imagens da TCBC ajudam a identificar reabsorções radiculares relacionadas, em dentes antagonistas, mesmo sendo localizadas pela vestibular e pela lingual. (ERICSON \& KUROL, 2000).

\section{Síndromes}

A TCFC ajuda a diagnosticar e acompanhar pacientes com síndromes relacionadas a alterações nos dentes, ossos e morfologia (OENNING et al., 2018).

Devido à menor dose de radiação emitida, em comparação com TCMD, TCFC é particularmente útil para monitorar exceções craniofaciais, pode haver várias anormalidades dentárias ou impactações dentais presentes (DAMASCENO et al., 2014).

\section{Doenças periodontais}

Não existem estudos sobre o uso da TCFC para o diagnóstico de doença periodontal em crianças e adolescentes (WOELBER et al., 2018).

Evidências encontradas em ensaios clínicos em adultos sugerem que a TCFC é usada para diagnosticar doença periodontal em crianças e adolescentes (Horner et al., 2020). A avaliação periodontal só deve ser realizada em situações específicas mais complexas, o que é extremamente raro em situações pediátricas (OENNING et al., 2018).

\section{Fenda orofacial}

Crianças com lábio leporino estão sofrendo exposição vitalícia à radiação e necessita de inspeção mais precisa; para este fim, a TCFC pode ser usada para: plano de cirurgia, monitoração do desenvolvimento de dentição adjacente, 
acompanhamento da cicatrização após o enxerto ósseo, verificação de fissuras residuais após enxerto (EUROPEAN COMISSION, 2012; HORNER et al., 2020; OENNING et al. 2018; WÖRTCHE et al., 2006).

Imagens tridimensionais aumentam a previsibilidade, além de reduzir a cirurgia, o tempo e os custos envolvidos (CHOI et al., 2012).

\section{Planejamento ortodôntico}

Em alguns casos clínicos, na elaboração de um tratamento ortodôntico, as avaliações da posição dental são recomendadas e precisas para evitar uma futura locomoção dos elementos dentários, que poderá levar a severas reabsorções dentárias e ósseas. A tomografia computadorizada auxilia na avaliação da espessura óssea vestibular e lingual (CONSOLARO, 2007).

Tomografia computadorizada é uma ótima ferramenta que auxilia na elaboração da terapêutica ortodôntica (GARIB, 2007).

De acordo com Consolaro, para adquirir imagens tomográficas em planos ortodônticos que permitam a avaliação, mais precisamente, da relação entre a crista óssea alveolar e o dente, a altura, a forma e o contorno da área do pescoço ao longo de toda a circunferência afirma:

Adquirir imagens tomográficas de volume em planos ortodônticos permitem avaliação, ainda mais precisamente, é a relação entre a crista óssea alveolar e o dente, a altura, a forma e o contorno da área do pescoço ao longo de toda a circunferência. Da mesma forma, o formato da raiz, especialmente a ponta da raiz e a relação coroa-raiz foram avaliados em detalhes. Os fatores mencionados neste parágrafo são a base para prever a reabsorção dentária para a adoção de um plano ortodôntico defensivo (CONSOLARO, 2007, p. 110).

\section{Discussão}

Segundo a literatura, a tomografia computorizada de feixe cônico reflete a maior precisão e detalhamento dos resultados obtidos em seu método de aquisição de imagens. Isso ajuda a planejar melhor as intervenções cirúrgicas, pois permite a visualização da estrutura em 3D (três dimensões). Terra et al. (2011) defenderam a afirmação anterior e trouxeram seus trabalhos, ou seja, a TCFC elimina defeitos de visualização bidimensional e traduz em radiografias tradicionais para auxiliar no diagnóstico mais preciso.

Conforme afirmado pelo autor acima, a tomografia computadorizada de feixe cônico permite a análise de imagens em três dimensões. Em relação às diferenças entre CBCT e tomografia computadorizada tradicional existem alguns pontos que indicam ser uma técnica alternativa mais interessante. Esta afirmação é encontrada nos trabalhos de Lima et al. (2009) e (2010), indicando que essa tecnologia foi criada especialmente na área odontológica.

Moura et al. (2018) traz algumas vantagens consistentes em relação á tecnologia discutida, como tomografia de feixe cônico com tomógrafo menor, possui uma menor dose de radiação e baixo custo para o paciente e fornece informações superior a TC. Além de ser um exame preciso, por não apresentar distorção, o exame é 1:1 onde é possível observar o tamanho, volume e a dimensão da lesão.

No que diz a respeito á aplicação clínica ela se destina a diversas áreas da odontologia, principalmente cirurgia, implantologia, ortodontia, endodontia, periodontia, distúrbio temporomandibular. Devido à falta de indicações e especificidade, a tecnologia CBCT é propícia para profissões que não eram reconhecidas anteriormente pela TC.

Uma das desvantagens da TCFC é que requer uma melhor técnica, o profissional tem que estar apto a usar o aparelho, ou seja, ter um conhecimento amplo do manuseio da técnica é um exame imagiológico de alto custo. 


\section{Considerações Finais}

Nesse estudo, foi abordada a utilização da tomografia computadorizada de feixe cônico como um método de diagnóstico eficaz e aliado na odontologia para complementar uma hipótese de diagnóstico, se destacando na odontopediatria, podendo ser solicitada para diversos casos clínicos, identificar lesões tais como as fraturas verticais, para o planejamento ortodôntico, para fendas orofaciais, síndromes genéticas, reabsorções radiculares, dentes impactados e traumas dentais. O cirurgião dentista deve ter o conhecimento prático e conhecer as técnicas, para saber analisar tais resultados, e saber ao certo o que deve observar, para definir um correto diagnóstico.

Pode-se concluir que a tomografia computadorizada ainda é um obstáculo para os cirurgiões-dentistas, entretanto, cabe ao cirurgião-dentista estar apto para buscar mais conhecimento sobre o assunto. É de extrema importância que os cirurgiões-dentistas se aprofundem mais no estudo das indicações da tomografia computadorizada de feixe cônico avaliando seus riscos, suas indicações e suas limitações. Desta forma também se faz fundamental a utilização da técnica empregada com precisão, além da colaboração do paciente, tornando assim um estudo discutido pela comunidade cientifica.

\section{Referências}

ABRAHAMS, J.J. The role of diagnostic imaging in dental implantology. Radiol Clin North Am 1993; 31(1):163-80.

ABRAMOVITCH, K. Pratical imaging of the temporomandibular joint. Tex Dent J 1995; 112(2):45-51.

ADA. The use of cone-beam computed tomography in dentistry: an advisory statement from the American Dental Association Council on Scientific Affairs. $J$ Am Dent Assoc. v.143:899-902. 2012.

AMERICAN ACADEMY OF PEDIATRIC DENTISTRY. Prescribing dental radiographs for infants, children, adolescents, and individuals with special health care needs. The Reference Manual of Pediatric Dentistry. Chicago, Ill: American Academy of Pediatric Dentistry. v.248-51.2020.

APS, J.K.M. Cone beam computed tomography in paediatric dentistry: overview of recent literature. European Archives Of Paediatric Dentistry, [S.L.], v.14, n.3, p.131-140, 6 abr.2013. Springer Science and Business Media LLC.

BARBOSA, J. et al. Aplicações da tomografia computadorizada na odontopediatria. Revista do Cromg. v.16, n. 2, 4 out. 2018.

BERNARDES, R.A. Estudo comparativo entre as tomografias computadorizadas 3D, ortopantomográficas e radiografias periapicais no diagnóstico de lesões periapicais, fraturas radiculares e reabsorções dentais. 2007. Tese (Doutorado em Endodontia) - Faculdade de Odontologia de Bauru, Universidade de São Paulo, Bauru, 2007.

BERNI, N.R.C. et al. Estudo da ocorrência de alterações morfológicas ou degenerativas da ATM utilizando a tomografia linear. Rev Assoc Bras Reab Oral. 4(2):81-4.2003.

BONTRAGER, K.L. Tratado de técnica radiológica e base anatômica. Rio de Janeiro: Guanabara-Koogan. 805p.2003.

CARRASCO, M.A. et al. Guías sobre el uso de tomografía computarizada de haz cónico en la evaluación pre-quirúrgica en implantología. Avances en Odontoestomatología, Madrid. v.34, n.4, p.183-192, ago.2018.

CHOI, H.S. et al. Influence of the alveolar cleft type on preoperative estimation using 3D CT assessment for alveolar cleft. Arch Plast Surg 39:477-482.2012.

CONSOLARO, A. FREITAS, P.Z. Tomografia Volumétrica (odontológica) versus helicoidal (médica) no planejamento ortodôntico e no diagnóstico das reabsorções dentárias. Rev. Clín. Ortodon. Dental Press Maringá. 6(4). 108- 11.2007.

D'ADDAZIO, P.S.S. et al. A comparative study between cone-beam computed tomography and periapical radiographs in the diagnosis of simulated endodontic complications. Int Endod J. 44:218-24.2011.

DAMASCENO, J.X. et al. Generalized odontodysplasia in a 5-year-old patient with Hallermann-Streiff syndrome: clinical aspects, cone beam computed tomography findings, and conservative clinical approach. Oral Surg Oral Med Oral Pathol Oral Radiol. 118(2):e58-e64.2014.

DURACK, C. PATEL, S. DAVIES, J, WILSON, R, MANNOCCI, F. Diagnostic accuracy of small volume cone beam computed tomography and intraoral periapical radiography for the detection of simulated external inflammatory root resorption. Int Endod J. 44(2):136-47.2011.

ERICSON, S, KUROÇ J. Incisor root resorptions due to ectopic maxillary canines imaged by computerized tomography: a comparative study in extractec teeth. Agle Orthod. 70:276-283.2000.

GARIB, D.G. et al. Tomografia Computadorizada de feixe cônico (Cone Beam): entendendo este novo método de diagnostico por imagem com promissora aplicabilidade na Ortodontia. R Dental Press Ortodon Ortop Facial. 12(2):139-56.2007.

HANEY, E. et al. Comparative analysis of traditional radiographs and cone beam computed tomography volumetric images in the diagnosis and treatment planning of maxillary impacted canines. Am J Orthod Dentofacial Orthop. 137:590-7.2010. 
HORNER, K, BARRY, S, DAVE, M. et al. Eficácia diagnóstica da tomografia computadorizada de feixe cônico em odontopediatria: uma revisão sistemática. Eur Arch Paediatr Dent 21. 407-426.2020.

KAMBUROG ${ }^{\sim}$, L.U.K, BARENBOIM, S.F, KAFFE. Comparison of conventional film with different digital and digitally filtered images in the detection of simulated internal resorption cavities - an ex 45 vivo study in human cadaver jaws. Oral Surg Oral Med Oral Pathol Oral Radiol Endod. 105:790-7.2008.

KAPILA, S.D, NERVINA, J.M. CBCT in orthodontics: assessment of treatment outcomes and indications for its use. Dento maxillo facial radiology, Erlangen. v.44, n.1,p.20140282.2015.

KATHERIA, B.C. et al. Effectiveness of impacted and supernumerary tooth diagnosis from traditional radiography versus cone beam computed tomography. Pediatr Dent. 32(4):304-9.2010.

KING, J.M, CALDARELLI, D.D, PETASNICK, J.P. DentaScanTM: a new diagnostic method for evaluating mandibular and maxillary pathology. Laryngoscope. 4(102):379-87.1992.

KOCHE, J. C. (2011). Fundamentos de metodologia científica.

KUMAR, V. et al. Comparison between cone-beam computed 49 tomography and intraoral digital radiography for assessment of tooth root lesions. Am $J$ Orthod Dentofacial Orthop. 139(6):e533-41.2011.

LIEDKE, G.S. et al. Influence of voxel size in the diagnostic ability of cone beam 48 tomography to evaluate simulated external root resorption. $J$ Endod. 35(2):233-5.2009.

LIMA, I.L.A, et al. Radiografias convencionais e tomografia computadorizada cone-beam para localização de dentes inclusos: relato de caso. Arq bras odontol. v.52, 58-64.2009. Disponível em: http://periodicos.pucminas.br/index.php/Arquivobrasileirodontologia/article/view/11 57.Acesso em: 29.09.2021.

LIMA, R.K.P, et al. Diagnóstico e planejamento em cirurgia parendodôntica: utilização da tomografia cone beam. RSBO (Online), v.7, n.4, p.474-480.2010. Disponível em: http://revodonto.bvsalud.org/scielo.php?pid=S1984- 56852010000400015\&script=sci_abstract. Acesso em: 29.09 .2021

MANSINI, R. et al. Utilização da tomografia computadorizada no diagnóstico de fraturas radiculares verticais. RGO. v.58, n.2, p.185-190.2010.

MOURA, J.R, et al. Aplicabilidade da tomografia computadorizada cone beam na odontologia. Rev. Odontol. Araçatuba (Impr.).39(2): 22-27, maio/ago.2018. Disponível em: https://pesquisa.bvsalud.org/portal/resource/pt/biblio913471. Acesso em: 29.09.2021.

OENNING, A.C. et al. DIMITRA Research Group, CT in paediatric dentistry: DIMITRA project position statement. Pediatr Radiol. 48:308-16.2018.

OLMEZA, H, GORGULUBS, AKINA, E. Measurement accuracy of a computer-assisted threedimensional analysis and a conventional two dimensional method.Angle Orthodontist. 2011.

PARKS, E.T. Computed tomography applications for dentistry. Dent Clin North Am.44(2):371-94.2000.

PATEL, S. et al. The potential applications of cone beam computed tomography in the management of endodontic problems. International Endodontic Journal, 40, 818- 830, 2007.

PRIMO, B.T., et al. Dentes retidos: novas perspectivas de localização. Rev. RFO, Passo Fundo. v.16, n.1, p.95-99, jan./abr.2011.Disponível em: http://files.bvs.br/upload/S/1413-4012/2011/v16n1/a2140.pdf. Acesso em 30/09/2021.

TERRA, G.T.C, et al. Tomografia Computadorizada Cone Beam: Avaliando sua precisão em medidas lineares. Journal of Biodentistry and Biomaterials. v.2, p.10-16, 2011. Disponível em: https://www.researchgate.net/publication/322068783_TE RRA_G_T_C_Oliveira_J_X_ DOMINGOS_V_B_T_C_ROSSI_JUNIOR_R_Tomografia_Computadorizada_Cone_Bea m_Avaliando_sua_precisao_em_m edidas_lineares_Journal _of_Biodentist y_and_Biomaterials_v_2_p_10-16_2011. Acesso em: 29/09/2021.

VIDIGAL, B.C.L, et al. Uso da tomografia Cone Beam na avaliação de fraturas radiculares. Rev. bras. odontol. Rio de Janeiro, v.71, n.2, p.152-5, jul./dez.2014. Disponível em: http://revodonto.bvsalud.org/pdf/rbo/v71n2/a08v71n2.pdf. Acesso em 30/09/2021.

WOELBER, J.P. et al. Accuracy and usefulness of cbct in periodontology: a systematic review of the literature. Int J Periodontics Restor Dent. 38:28997.2018.

WÖRTCHE, R. et al. Clinical application of cone beam digital volume tomography in children with cleft lip and palate. Dentomaxillofac Radiol. 35(2):8894.2006. 